\title{
Time and Finance Consumption in Reaching Out for Neurosurgery Consultant in Outpatient Department
}

\author{
Sandeep Mohindra ${ }^{1}$ Avondeep Dhaliwal ${ }^{2}$ \\ ${ }^{1}$ Department of Neurosurgery, Postgraduate Institute of Medical \\ Education and Research, Chandigarh, India \\ 2 Division of Neurology and Neurosurgery Nursing, National Institute \\ of Nursing Education, Postgraduate Institute of Medical Education \\ and Research, Chandigarh, India \\ Indian J Neurosurg 2015;4:22-25.
}

Address for correspondence Avondeep Dhaliwal, MSc, Division of Neurology and Neurosurgery Nursing, National Institute of Nursing Education, Postgraduate Institute of Medical Education and Research, Chandigarh, India (e-mail: avondhaliwal89@gmail.com).

\begin{abstract}
Background Follow-up after surgical intervention is a vital part of patient safety and it also ensures that patients with chronic conditions receive the appropriate secondary care input.

Aims and Objective To calculate the time and finance patient spends in reaching out to the clinician. To outline the expenditure incurred on the patients' attendants, who travel along with the patient.

Methods A prospective study was conducted on study subjects $(N=103)$ who visited OPD Department of Neurosurgery, PGIMER, Chandigarh, India. The researcher collected details directly from the patients or from their closest available attendants by using prepared questionnaire.

Results The mean time spent by patients in reaching OPD complex was 9 hours, in reaching consultant chamber after reaching OPD building was 3 hours, and for standing in queue just to get their registration done was 45 minutes. The mean time spent waiting outside for treating doctor was 60 minutes and for meeting doctor faceto-face was 10 minutes. A total of 26 patients came for follow-up without attendants and they spent a mean of INR 115. Attendants came with 77 patients and mean

\section{Keywords}

- time and finance consumption

- neurosurgery consultant

- outpatient department expenditure on their transportation was INR 484.

Conclusion Significant time wasted by patients for registration of OPD cards can be minimized by making appointment system by using phone call or the internet. Limitation of time consumption and accuracy of data recording can also be enhanced by installing Hospital Information System (HIS) and making OPD paperless. Cost-cutting methods can include stringent criteria for calling unconscious or dependent patients for follow-up in more judicious fashion.
\end{abstract}

\section{Introduction}

Follow-up after surgical intervention for patients is mandatory to gauge the ongoing improvement in clinical outcome and day-to-day activities and also to make note of any deterioration while in convalescence period postoperatively. Follow-up is a vital part of ongoing patient safety. It allows for subsequent investigations to be checked and acted upon, encourages specialist review of patients, and ensures that patients with chronic conditions receive the appropriate secondary care input. ${ }^{1}$ Follow-up of surgically operated patients highly depends upon the discharge summaries the patients carry along. These may be handwritten, typed, or computerized and digitalized in electronic forms on the internet. These discharge summaries are helpful in getting a better record of follow-up and increasing the reliability of punctual follow-up. ${ }^{2}$ published online March 20, 2015
DOI http://dx.doi.org/ $10.1055 / \mathrm{s}-0035-1549064$ ISSN 2277-954X. (c) 2015 Neurological Surgeons' Society of India
License terms

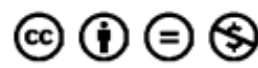


This study aims to highlight and quantify the current problems of time and financial resources spent by patients and their attendants in reaching out to his clinician. Indirectly, the study would outline the financial liability on the patients' attendants also, who need to travel along with the patient.

\section{Materials and Methods}

The study was conducted using quantitative, descriptive prospective survey approach. Study subjects were conveniently selected from all follow-up patients visiting OPD complex at neurosurgery department, PGIMER, Chandigarh (tertiary care hospital, with bed strength over 1,780 ), who were willing to participate in the study and can understand Hindi, Punjabi, or English. A total of 103 subjects in OPD follow-up after neurosurgical interventions, including both cranial and spinal cases, were included. Patients who required opinion from multiple specialties or requiring investigations like hematological or radiological scans were not a part of this study. All patients staying at distance from their native homes just for treatment purposes were not a part of the study.

A questionnaire was prepared to collect the data. It consists of the following components: (1) demographic data sheet that has items dealing with demographic characteristics and (2) questionnaire to assess time and finance consumption in reaching out for neurosurgery consultant. It includes questions related to patient status, mode of transport, place of starting, date of starting, time of starting, and financial resources spent to reach OPD for consultation.

Demographic data including age, sex, place of origin of patients, and place of residential address including contact mobile numbers were noted. Patient status including the ability to move, wheelchair bound, or bedridden was noted. Patient status of consciousness was also noted. Mode of transport, classified into three types, namely, public transport, private personal, or hired private vehicle, was documented for all. Details included patient's place of starting, time of starting from home, time of reaching OPD complex, time spent while standing for registration, time spent while waiting to get the opinion of treating clinician, total time given by clinician to the patient, and finally total time in reaching back to residence. Additional information was drawn regarding financial resources spent to reach OPD for clinician's consultation.

Study subjects were enrolled on the basis of inclusion criteria (mentioned earlier). Patients were enrolled from two OPDs (dated January 11-18, 2014) over a span of complete day, starting at 8 Am till 3 PM. All OPD patients were part of the study and the details were noted directly from the patients or from their closest available attendants.

\section{Results}

-Table 1 depicts that most of the follow-up patients belonged to the fifth decade of life ( $n=49,47.6 \%)$, while least number of patients belonged to the seventh decade of
Table 1 Sociodemographic profile of study subjects $(N=103)$

\begin{tabular}{|c|c|}
\hline Personal characteristics & $n(\%)$ \\
\hline \multicolumn{2}{|l|}{ Age (y) } \\
\hline $0-10$ & $08(7.8)$ \\
\hline $11-20$ & $05(4.8)$ \\
\hline $21-30$ & $06(5.8)$ \\
\hline $31-40$ & $11(10.6)$ \\
\hline $41-50$ & $49(47.6)$ \\
\hline $51-60$ & $20(19.4)$ \\
\hline $61-70$ & $04(3.8)$ \\
\hline \multicolumn{2}{|l|}{ Gender } \\
\hline Male & $64(62)$ \\
\hline Female & $39(38)$ \\
\hline
\end{tabular}

life $(n=4,3.9 \%)$. A total of $62 \%$ were males and $38 \%$ were females.

- Table 2 depicts that majority of patients were ambulatory, while $11 \%$ patients were bedridden and were brought on trolleys. A total of $8 \%$ of patients were wheelchair bound and required assistance for ambulation. Most of the patients were fully conscious (91\%), whereas rest of the patients were unconscious (9\%).

- Table 3 depicts that public transport like buses, trains, and auto rickshaws were utilized by majority (79.6\%) of follow-up patients, while $11.6 \%$ patients hired private vehicles like taxicabs for reaching their destinations. Only $8.7 \%$ patients came by personal private vehicles like personal cars or two-wheelers.

- Table 4 depicts that the mean time taken by patients for reaching OPD complex was 9 hours; the mean time taken for

Table 2 Distribution of study subjects according to health status $(N=103)$

\begin{tabular}{|l|l|}
\hline Patient status & $\boldsymbol{n}(\%)$ \\
\hline Ambulatory & $84(81)$ \\
\hline Wheelchair bound & $08(8)$ \\
\hline Bedridden & $11(11)$ \\
\hline Conscious & $94(91)$ \\
\hline Unconscious & $09(9)$ \\
\hline
\end{tabular}

Table 3 Distribution of study subjects according to mode of transport used for reaching OPD complex $(N=103)$

\begin{tabular}{|l|l|}
\hline Mode of transport & $\boldsymbol{n}(\%)$ \\
\hline Public transport & $82(79.6)$ \\
\hline Private personal & $09(8.7)$ \\
\hline Hired private & $12(11.6)$ \\
\hline
\end{tabular}


Table 4 Time consumption in reaching for neurosurgery consultant $(N=103)$

\begin{tabular}{|l|l|l|}
\hline Event & Mean time spent & Range of time spent \\
\hline From residence to doctor & $9 \mathrm{~h}$ & $4-48 \mathrm{~h}$ \\
\hline Hospital main gate to doctor & $3 \mathrm{~h}$ & $1-6 \mathrm{~h}$ \\
\hline Time spent in queue for card registration & $45 \mathrm{~min}$ & $15 \mathrm{~min}$ to $3 \mathrm{~h}$ \\
\hline Waiting period outside doctor's chamber & $1 \mathrm{~h}$ & $15 \mathrm{~min}$ to $4 \mathrm{~h}$ \\
\hline Time duration of doctor-patient face-to-face meeting & $10 \mathrm{~min}$ & $2-30 \mathrm{~min}$ \\
\hline Total time spent for OPD visit & $18 \mathrm{~h}$ & $8-96 \mathrm{~h}$ \\
\hline
\end{tabular}

Table 5 Finance consumption in reaching for neurosurgery consultant $(N=103)$

\begin{tabular}{|l|l|l|}
\hline Financial burden & Mean & Range \\
\hline Patient visiting OPD alone $(n=26)$ & INR 115 & INR 50-2,000 \\
\hline Attendant expenditure $(n=77)$ & INR 484 & INR 25-7,200 \\
\hline
\end{tabular}

reaching consultant chamber after reaching OPD building was 3 hours. The mean time spent by patients standing in queue just to get their registration done was 45 minutes and the mean time spent waiting for treating doctor was 60 minutes, while the mean time spent face-to-face with treating doctor was 10 minutes.

- Table 5 depicts that 26 patients came for follow-up without any attendants and they spent a mean of INR 115, while 77 patients came along with attendants and the mean expenditure incurred on their transportation was INR 484.

\section{Discussion}

As the OPD follow-up was taken from neurosurgical patients, majority belonged to the fifth decade of life. Neurosurgical procedures and disorders render a huge number of patients bodily disabled. So, gathering data regarding their physical status becomes of utmost importance, as it has an important bearing on time taken by these patients to reach OPD. Ambulatory patients tend to spend less time in reaching OPD when compared with bedridden patients, and similarly unconscious patients would require more time in reaching for their follow-up visits. The mode of transport was public transport for 82 patients, which obviously contributed to more time consumption for OPD follow-up.

Patients spent a huge amount of time in reaching their doctors, which was as high as 48 hours for one patient, while locally residing patients could reach their doctors in 4 hours. Still, the mean time taken by patients was 9 hours. This massive time of transportation is attributed to the huge distances or far-off places these patients come from for their follow-ups. The mean time spent within the hospital only for fulfilling all the paperwork formalities was 3 hours. The patients spent a mean of 45 minutes standing in queue to get their card registered. This significant waste of time is mainly due to absence of any system of appointment fixation by telephone or the internet. Further, there is no fax system by which patient records can be assessed by the doctor in digitalized manner, causing more wastage of time. The patients carry handwritten discharge summaries and not the computer printout summaries, which leads to difficulty in interpreting the exact registration numbers of patients, thereby increasing queue timings. The waiting time of patient outside OPD chambers ranged from 15 minutes to 4 hours (mean $=60$ minutes). This timing can also be curtailed if prior appointment schedules are done via phone call or Web sites. Scheduling appointment system would drastically cut down patient waiting time and rush during specific hours of OPD timing and would help to scatter all patients uniformly over the whole day.

As 26 patients had come alone without attendant, expenditure on transportation remained meager and was just INR 115 (mean), but this was at the cost of huge time that patients wasted in reaching for follow-ups using public transport systems. The addition of attendants increased the cost from INR 25 to INR 7,200, putting a huge financial burden on already physically disabled patient. This particular cost is specific for neurosurgical patients and sums up travelling costs by huge amounts. Hence, OPD follow-ups for such patients should be meticulously charted out and planned, so as to minimize this additional financial burden.

\section{Conclusion}

As majority of patients utilize public transport system to reach their doctors for follow-ups, a lot of time is consumed for transportation. Significant time is wasted by patients for administrative jobs like registration of OPD cards, and this can be minimized by making appointment system mandatory by using phone call or the internet. Limitation of time consumption and accuracy of data recording can also be enhanced by installing HIS and making OPD paperless. Token system can be put to use, so 
as to make patients comfortable while waiting for their turns. Digital display of patients' turn outside doctors' chamber also makes time to be utilized more efficiently. Cost-cutting methods can include stringent criteria for calling unconscious or dependent patients for follow-up in more judicious fashion.

\section{References}

1 Wimble K, Yeong K. Improving patient follow-up after inpatient stay. BMJ Qual Improv Report 2012;1: 10.1136/bmjquality.u474. w148

2 Barr R, Chin KY, Yeong K. Improving transmission rates of electronic discharge summaries to GPs. BMJ Qual Improv Report 2013;2: 10.1136/bmjquality.u756.w1013 